\title{
Avaliação e intervenção para a reabilitação cardiopulmonar de pacientes recuperados da COVID-19*
}

Evaluation and intervention for cardiopulmonary rehabilitation of COVID-19 recovered patients*

CACAU, Lucas de Assis Pereira ${ }^{1}$; MESQUITA, Rafael²; FURLANETTO, Karina Couto ${ }^{3}$; BORGES, Daniel Lago Silva"; FORGIARINI JR., Luíz Alberto ${ }^{5}$; MALDANER, Vinícius ${ }^{6}$; SOUZA, Yves $\mathrm{de}^{7}$; CIPRIANO JR., Gerson ${ }^{8}$; CARVALHO, Celso ${ }^{9}$; NOGUEIRA, Ingrid Correia ${ }^{10}$; TOMAZI, Laura $^{11}$; KARSTEN, Marlus ${ }^{12}$; em nome do Comitê COVID-19 da ASSOBRAFIR.

\footnotetext{
Resumo

Com a pandemia de COVID-19, é necessário o rápido entendimento das prováveis e graves sequelas que os pacientes sobreviventes podem desenvolver, assim como torna-se urgente traçar planos de ação para enfrentar tal situação desde o processo de alta hospitalar até a inserção em serviços de reabilitação cardiopulmonar. De acordo com recomendações internacionais, uma avaliação individualizada deve ser realizada e documentada no momento da alta, a qual deve contemplar as necessidades imediatas (controle dos sintomas como dispneia, fadiga e dor), assim como as necessidades a curto e médio prazo (melhora da função física e emocional; retorno ao trabalho; dentre outros). É aconselhável que nas primeiras semanas após a alta, o paciente seja atendido

* Revisado por membros do Comitê COVID-19 da ASSOBRAFIR, nomeado por meio do memorando Nº03/2020.

1 Clínica Intervent, Aracaju - SE, Brasil. Email: luccacau@gmail.com. LAPC - https://orcid.org/0000-0002-2676-7854

2 Departamento de Fisioterapia, Universidade Federal do Ceará (UFC), Fortaleza, Ceará, Brasil. RBM - https://orcid.org/0000-0002-8048-3393

3 Programa de Pós-Graduação em Ciências da Reabilitação, Universidade Pitágoras-Unopar (UNOPAR), Londrina, Paraná, Brasil. KCF - https://orcid.org/0000-0002-7496-7228

4 Hospital Universitário da Universidade Federal do Maranhão. DLSB - https://orcid.org/0000-0003-4082-527X

5 Programa de Pós graduação em Saúde e desenvolvimento humano da Universidade La Salle, Porto Alegre, RS.

LAFJ - https://orcid.org/0000-0002-6706-2703

6 Programa de Pós graduação em movimento humano e reabilitação- Unievangelica, Anápolis, GO.

VM - https://orcid.org/0000-0002-7804-7517

7 Universidade Veiga de Almeida (UVA); Departamento de Pneumologia, Universidade do Estado do Rio de Janeiro (UERJ). YS - https://orcid.org/0000-0001-5868-8360

8 Universidade de Brasília, DF. GCJ - https://orcid.org/0000-0001-6323-7003

9 Faculdade de Medicina, Universidade de São Paulo, SP. CS - https://orcid.org/0000-0003-3046-3412

10 Docente do Curso de Fisioterapia e do Programa de Pós-graduação em Tecnologia Minimamente Invasiva e Simulação na Área da Saúde (TEMIS) do Centro Universitário Christus - Unichristus. ICN - https://orcid.org/0000-0002-8711-817X

11 Laboratório de Avaliação e Reabilitação das Disfunções Cardiovascular, Oncológica e Respiratória (LACOR), Programa de PósGraduação em Ciências do Movimento Humano (PGCMH), Instituto de Ciências da Saúde (ICS), Universidade Federal do Pará (UFPA). Complexo Hospitalar da Universidade Federal do Pará (CH-UFPA). LM - https://orcid.org/0000-0002-3115-2571

12 Departamento de Fisioterapia e Programa de Pós-Graduação em Fisioterapia, Universidade do Estado de Santa Catarina (UDESC), Florianópolis, Santa Catarina, Brasil; Programa de Pós-Graduação em Ciências da Reabilitação, Universidade Federal de Ciências da Saúde de Porto Alegre (UFCSPA), Porto Alegre, Rio Grande do Sul, Brasil.

MK - https://orcid.org/0000-0002-2476-7981
} 
através de comunicação digital, a qual deve incluir imagem e áudio por questões de segurança. Componentes mandatórios dos programas de reabilitação cardiopulmonar incluem exercícios de força e resistência, além do trabalho da musculatura inspiratória. A abordagem educacional, é item importante no processo de tratamento. A reabilitação de doentes críticos acometidos por COVID-19 após alta hospitalar é de fundamental importância, especialmente naqueles que evoluíram com o quadro grave da doença, e que necessitaram de internação em UTI.

Palavras-chave: Fisioterapia; Reabilitação cardiopulmonar; COVID-19.

\section{Abstract}

With the pandemic of COVID-19, it is necessary to quickly understand the probable and serious sequelae that surviving patients may develop, as well as it is urgent to draw up action plans to face this situation, which begins from the discharge process until insertion in cardiopulmonary rehabilitation services. According to international recommendations, an individualized assessment must be performed and documented at the time of discharge, which must include immediate needs (control of symptoms such as dyspnoea, fatigue and pain), as well as short and medium term needs (improvement of physical and emotional function; return to work; among others). It is recommended that in the first weeks after discharge, supervision occurs through digital communication, which must include image and audio for security reasons. Mandatory components of cardiopulmonary rehabilitation programs include strength and endurance exercises, in addition to the inspiratory muscles work. The educational approach is also an important item in the treatment process. The rehabilitation of critically ill patients affected by COVID-19 after hospital discharge is of interest, especially in those who developed the severe condition of the disease or required ICU admission.

Keywords: Physiotherapy; Cardiopulmonary rehabilitation; COVID-19.

\section{Objetivo}

O objetivo do presente documento é apresentar as informações científicas atualmente disponíveis, a fim de facilitar a reabilitação de pacientes acometidos pela COVID-19, especialmente daqueles que evoluíram com o quadro grave da doença e que normalmente necessitaram de internação em Unidade de Terapia Intensiva (UTI).

\section{Contextualização}

Os pacientes com COVID-19 podem desenvolver acometimentos graves durante a hospitalização tais como insuficiência respiratória, choque séptico e/ou disfunção de múltiplos órgãos levando à necessidade de oxigênio suplementar e ventilação mecânica prolongada ${ }^{4}$. Algumas comorbidades estão associadas a um pior prognóstico, dentre elas: idade ( $>65$ anos), doença pulmonar obstrutiva crônica (DPOC), asma, cardiopatias (hipertensão arterial severa), doença cerebrovascular, diabetes, insuficiência renal e câncer ${ }^{5,6}$. Entre os sintomas clássicos estão a febre, dispneia e tosse seca. Porém, alguns pacientes podem apresentar congestão nasal, cefaleia, dor de garganta, mialgia, hipogeusia (perda de paladar), hiposmia (perda de olfato), conjuntivite, diarreia, vômito ou erupção cutânea. $\mathrm{Na}$ região da Lombardia, que foi um dos epicentros da doença na europa, a mortalidade entre os pacientes que necessitaram de internação em UTI chegou a 26\% ${ }^{7}$. Já em Nova Iorque, que se tornou um dos epicentros na américa do norte, a mortalidade entre os pacientes que necessitaram de ventilação mecânica chegou a $88,1 \%{ }^{7,8}$.

A maioria dos pacientes apresenta alterações na tomografia computadorizada (TC) do tórax ${ }^{9}$. A infecção viral pode aumentar o risco de fibrose pulmonar, que pode se formar durante o processo 
cicatricial da inflamação crônica pulmonar ou doenças proliferativas, com substituição gradual dos componentes celulares pelos tecidos da cicatriz ${ }^{10} ; 45 \%$ dos pacientes apresentaram sinais de fibrose pulmonar dentro de um mês após a infecção por SARS-CoV e desenvolveram fibrose pulmonar de 3 a 6 meses após a infecção ${ }^{8}$. Esses estudos sugerem que a fibrose pulmonar se tornará uma das sequelas em pacientes com infecção por COVID-19 ${ }^{11}$.

Pacientes com COVID-19 desenvolvem resposta inflamatória importante, a qual pode acarretar em inflamação vascular e causar alterações como miocardite e arritmias ${ }^{12}$. Durante a hospitalização, diversos estudos têm observado que um número considerável de pacientes acaba desenvolvendo alterações cardíacas e insuficiência hepática e renal dentre outras disfunções ${ }^{13-15}$, as quais também podem contribuir para pior capacidade física e funcional.

Considerando que muitos pacientes hospitalizados permanecem sob ventilação mecânica invasiva por tempo prolongado, necessitam de posição prona e fazem uso de sedação, bloqueador neuromuscular e/ou corticoides, acredita-se que muitos desses pacientes poderão apresentar alterações como a redução da força e da resistência da musculatura periférica e respiratória (relacionada a fraqueza muscular adquirida na UTI), do tônus muscular e da amplitude de movimento articular, bem como déficits de equilíbrio e coordenação motora dentre outras alterações. Os comprometimentos relacionados à internação em UTIs e sobre diferentes domínios de saúde tem recebido a denominação de síndrome pós-terapia intensiva, a qual provavelmente estará presente em muitos pacientes acometidos pela COVID-19. No entanto, ainda não se sabe qual a proporção de pacientes que evolui com alterações extrapulmonares, o que também justifica uma avaliação detalhada dessa população².

Dos poucos estudos já concluídos que abordam reabilitação cardiopulmonar nos pacientes após a COVID-19, um apresenta dados de capacidade funcional de exercício com uma distância percorrida no teste de caminhada de seis minutos (DTC6M) basal $<200 \mathrm{~m}$, além da presença de níveis significativos de ansiedade e depressão em pacientes que apresentam alterações intersticiais na TC de tórax ${ }^{16 .}$ Acredita-se ainda que os pacientes irão apresentar significativa fraqueza muscular após a alta hospitalar, o que inclui os músculos respiratórios devido ao período prolongado na UTI, maior tempo em ventilação mecânica e altas doses de sedativos e corticoides ${ }^{17,18}$. Além disso, muitos pacientes referem dispneia aos esforços e transtorno de estresse pós-traumático após a alta hospitalar ${ }^{16}$.

\section{Avaliação dos pacientes pós-COVID-19}

A avaliação desses pacientes pode ser feita em dois momentos distintos (Figura 1) e deve considerar diversos aspectos, incluindo: risco de contaminação do profissional e dos pacientes, e o local em que a avaliação será realizada. Inicialmente, durante a alta hospitalar deve-se verificar a necessidade de orientações para atividades domiciliares e de encaminhamento para serviços de reabilitação. Num segundo momento, já nos centros de reabilitação e após a saída do isolamento social, deve-se avaliar as limitações do paciente e direcionar a prescrição do programa de reabilitação. 
Figura 1 | Linha de cuidados nos distintos momentos pós-COVID-19. TC6: teste da caminhada de 6 minutos.

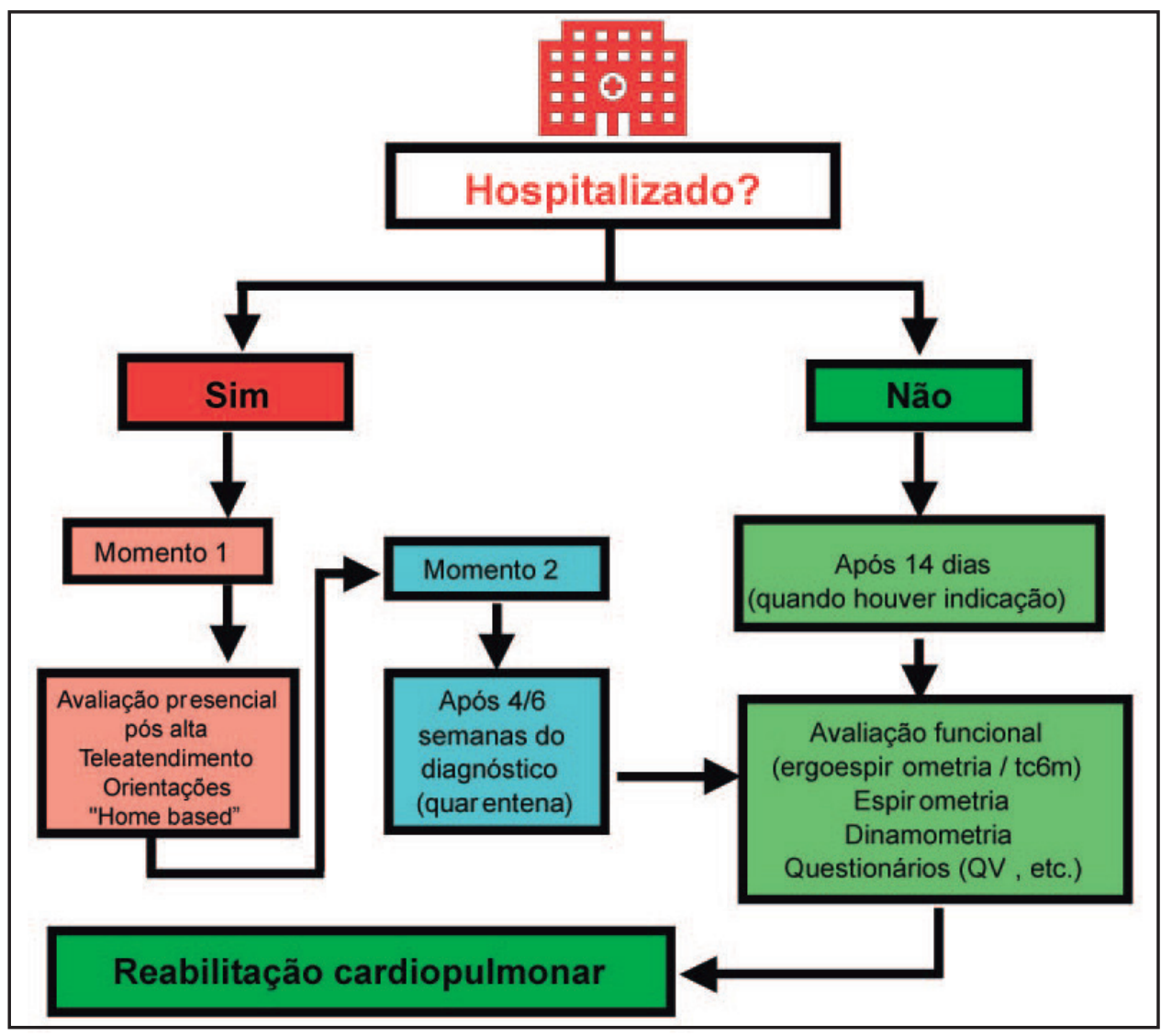

\section{Avaliação na alta hospitalar}

Devido ao grande número de internações durante a pandemia, muitos pacientes hospitalizados com COVID-19 provavelmente receberão alta precoce. No entanto, isso não significa que o paciente parou de transmitir a doença. Evidências científicas sugerem que o paciente com COVID-19 pode continuar transmitindo a doença mesmo após semanas da infecção e após os sintomas terem desaparecido ${ }^{19}$. Portanto, devido à escassez de testes clínicos para confirmar a presença do vírus bem como de evidências robustas que indiquem quando exatamente o paciente deixa de transmitir a doença, o fisioterapeuta deve tomar todos os cuidados para evitar a sua contaminação durante o processo de avaliação, como garantir a utilização de equipamentos de proteção individual (EPIs) adequados e uma higienização cuidadosa dos equipamentos utilizados ${ }^{20}$.

No momento da alta hospitalar, é importante registrar qualquer tipo de doença ou problema relevante desenvolvido durante o período de internação (p. ex., arritmias, miocardite), bem como doenças ou problemas relevantes apresentados antes da internação (p. ex., doença cardíaca prévia). Durante a aplicação de testes físicos, deve-se ter cuidado para evitar gasto energético excessivo, visto que pouco se sabe sobre os possíveis riscos de descompensação desses pacientes ${ }^{2}$. De acordo com recomendações internacionais, uma avaliação individualizada deve ser realizada e documentada no momento da alta, a qual deve contemplar:

Avaliação das necessidades imediatas: controle dos sintomas (p. ex., dispneia, fadiga e dor); necessidade de suplementação de oxigênio, nutrição e suporte psicológico e social adequados; mobilidade com segurança; dentre outros. 
Avaliação das necessidades a curto e médio prazo: melhora da função física e emocional; retorno ao trabalho; dentre outros.

Para as avaliações físicas no momento da alta é provável que equipamentos tecnologicamente avançados não estejam disponíveis, devido ao grande número de internações. Portanto, os fisioterapeutas podem utilizar testes simples e de rápida aplicação. A avaliação da perda de peso e massa muscular é de fundamental importância, bem como do impacto neuropsicológico causado pela COVID-19, o qual deve ser avaliados por uma equipe multiprofissional, idealmente. É importante ressaltar que, até o presente momento, a avaliação de função pulmonar e da capacidade máxima de exercício não são recomendadas nos pacientes sobreviventes de COVID-19 nas primeiras 6-8 semanas após a alta, devido ao potencial risco de contaminação dos avaliadores ou de outros pacientes que estejam presentes nos laboratórios de avaliação ${ }^{2}$. Entretanto, um estudo que realizou a avaliação da função pulmonar imediatamente após a alta, utilizando todos os cuidados para evitar a contaminação, identificou presença de alterações na capacidade de difusão de monóxido de carbono e na capacidade pulmonar total, especialmente em pacientes que evoluíram com o quadro mais grave da doença. Diferentemente das recomendações anteriores, esses autores sugerem que a função pulmonar seja avaliada após a alta caso existam condições seguras para a utilização da mesma ${ }^{21}$. Vale ressaltar que recentemente, um grupo de pesquisadores europeus desenvolveu uma escala para a avaliação do estado funcional de pacientes acometidos pela COVID19, a escala Post-COVID-19 Functional Status ${ }^{22}$. Essa é uma escala de fácil e rápida aplicação que pode ser útil para a avaliação longitudinal dos pacientes.

Assim, existem diversas ferramentas que podem ser utilizadas para avaliação da função cardiorrespiratória e neuromioarticular no momento da alta hospitalar. Nesta fase da avaliação, os autores do presente documento recomendam as ferramentas apresentadas no Quadro 1.

Quadro 1 | Ferramentas para avaliação cardiorrespiratória e neuromioarticular.

\begin{tabular}{|lc|}
\hline \multicolumn{1}{|c|}{ FUNÇÃo } & FERRAMENTA DE AVALIAÇÃo \\
\hline Força muscular periférica & Escore do MRC \\
Tônus muscular & Dinamometria de preensão palmar \\
Amplitude de movimento & Escala de Ashworth \\
Equilíbrio & Goniometria \\
Função física/mobilidade/estado & Escala de equilíbrio de Berg \\
funcional & Physical Function in Intensive care Test \\
& scored (PFIT-s) \\
& Functional Status Score for the ICU (FSS-ICU) \\
Intensive Care Unit Mobility Scale (IMS) \\
Post-COVID-19 Functional Status \\
Desempenho físico & Medida da Independência Funcional \\
& Teste de velocidade da marcha \\
& Teste de caminhada de 6 minutos \\
& Short Physical Performance Battery \\
& Timed up \& go \\
& Teste de sentar e levantar de 30 segundos \\
& ou 1 minuto \\
Força/função muscular respiratória & Manovacuometria \\
& Ultrassonografia \\
& Peak-flow \\
\hline
\end{tabular}




\section{Acompanhamento dos pacientes pós alta}

Cabe enfatizar que a COVID-19 é uma doença infecciosa com complicações potencialmente graves, cujo o impacto ainda não é totalmente compreendido. Dessa forma, faz-se necessário monitorar cuidadosamente os pacientes ao longo do processo de reabilitação ${ }^{11}$. Neste contexto, o telemonitoramento pode ser utilizado principalmente para pacientes de maior vulnerabilidade, ou seja, aqueles que sobreviveram o quadro grave da doença, devido ao alto risco de eventos cardiovasculares e ao aumento da mortalidade após a fase aguda da doença ${ }^{12}$, semelhante ao que acontece com pacientes que apresentaram exacerbação da DPOC ou pneumonia adquirida na comunidade ${ }^{19}$. Os fisioterapeutas deverão acompanhar esse grupo de risco com um plano de manejo adequado visando o reconhecimento imediato de complicações (p. ex., cardiovasculares), principalmente nos primeiros 30 dias após a resolução da fase aguda da doença. A mensuração de parâmetros como a saturação de pulso de oxigênio $\left(\mathrm{SpO}_{2}\right)$, a frequência cardíaca, a pressão arterial e a identificação de sinais como febre ou sintomas como a dispneia (avaliada por meio de ferramentas auto administráveis como a escala de Borg modificada ou a escala modificada do Medical Research Council - mMRC), são fundamentais para o reconhecimento do agravamento do quadro. A gravidade da COVID-19 tem sido associada à presença de dispneia, queda da $\mathrm{SpO}_{2}$ em ar ambiente (p. ex., <95\%) e hipotensão. A adesão ao telemonitoramento permitirá o acompanhamento dos pacientes nesse período crítico de transição até que o surto da doença seja contido, bem como permitirá aumentar o conhecimento sobre o manejo adequado da disfunção causada pela doença nos sobreviventes.

\section{Avaliação nos centros de reabilitação}

Considerando o término do isolamento social, pacientes sobreviventes de COVID-19 poderão ser avaliados e tratados em centros de reabilitação. Na escassez de evidências científicas específicas para a avaliação do paciente com COVID-19, recomenda-se que diretrizes de reabilitação pulmonar e cardíacas sejam utilizados como documentos norteadores, além de considerar as individualidades dos pacientes. Como conteúdo básico e com o objetivo de traçar o perfil funcional dos pacientes após hospitalização pela COVID-19 é importante realizar uma avaliação abrangente que inclua a avaliação de desfechos como a capacidade de exercício, através do teste de esforço cardiopulmonar (ergoespirometria) ou testes de campo com validade comprovada ${ }^{23}$; a força muscular periférica, para otimizar a prescrição da carga nos exercícios de força18; os níveis de atividade física na vida diária, por meio de pedômetros ou outros monitores de atividade física ${ }^{24-26} \mathrm{e}$; as atividade de vida diária, por meio de questionários ou avaliações objetivas ${ }^{27,28}$. De forma complementar, a avaliação de desfechos de saúde mental como a qualidade de vida relacionada à saúde, utilizando-se instrumentos genéricos ou específicos para pacientes com alterações respiratórias e/ou cardiovasculares, e a avaliação de sintomas de ansiedade e depressão ajudam na percepção de ganhos além das capacidades físico-funcionais. Além disso, a avaliação desses desfechos favorece a integralidade dos cuidados fisioterapêuticos, já que a maioria dos pacientes após a internação hospitalar em UTI apresentam limitações importantes ligadas a esses desfechos ${ }^{29}$. 


\section{Componentes da reabilitação cardiopulmonar}

\section{Teleatendimento}

As estratégias de teleconsulta e telemonitoramento já são comuns em vários países, com programas bem estabelecidos além de comprovada segurança desse método ${ }^{30}$. De forma excepcional e em caráter temporário o COFFITO, através da resolução no 516/2020, liberou a realização destas modalidades durante o tempo da pandemia do novo coronavírus16. Seguindo recomendações internacionais é aconselhável que nas primeiras semanas após a alta, o paciente seja atendido através de comunicação digital, a qual deve incluir imagem e áudio por questões de segurança ${ }^{2}$. É recomendável que nenhum tipo de avaliação ou intervenção sejam adaptados para o uso em teleconsulta de forma que configurações técnicas básicas que garantam sua segurança e efetividade sejam alteradas. Existem diversas avaliações e intervenções simples, com forte evidências e que podem ser reproduzidas em vídeochamada sem gerar riscos maiores aos pacientes ${ }^{31}$.

\section{Reabilitação ambulatorial}

O conhecimento sobre as alterações funcionais, incluindo as da função pulmonar, da capacidade de exercício, dos exames laboratoriais e de imagem, bem como a identificação da presença de comorbidades, são fatores fundamentais para auxiliar na indicação das estratégias de tratamento e na assertividade da prescrição das atividades terapêuticas.

Componentes mandatórios dos programas de reabilitação cardiopulmonar incluem exercícios de força e resistência, além do trabalho da musculatura inspiratória. A abordagem educacional, item importante do processo de tratamento, deve incluir técnicas de reeducação da respiração, técnicas de eliminação de secreções, benefícios da prática de exercícios e atividades físicas, técnicas de conservação de energia durante as atividades da vida diária, ingestão alimentar saudável e o que realizar nas atividades de lazer.

Mensurar e controlar a sintomatologia e a qualidade de vida relacionada à saúde, assim como as atividades de vida diária e os níveis de ansiedade e depressão são fundamentais para um processo de reabilitação integral, já que parte dos pacientes necessitaram de hospitalização, incluindo permanência na UTI, e esses desfechos impactam de forma importante na vida do paciente após a internação e durante o período de reabilitação.

Uma atenção especial após o acometimento pela COVID-19 deve ser direcionada aos pacientes portadores de doenças previas de origem respiratória, cardiovascular ou metabólica, principalmente na definição da intensidade do programa e das cargas e resistências dos exercícios utilizados.

Dimensionar o número de pacientes por grupo de acordo com normas de distanciamento e o tamanho do ambiente utilizado para reabilitação é condição essencial para se garantir a segurança dos profissionais e pacientes. Ainda, ressalta-se a importância de utilização de EPIs pelo fisioterapeuta e pelo paciente.

\section{Reabilitação domiciliar}

A reabilitação cardiopulmonar domiciliar ("home-based") é geralmente definida como um programa de exercícios não supervisionado, realizados fora do ambiente clínico ou hospitalar, e que pode utilizar aconselhamento por telefone, aplicativos para smartphones ou até mesmo por materiais impressos, todos nos sentido de repassar orientaçãoes ao paciente ${ }^{32-35}$. O Quadro 2 apresenta as 
vantagens e desvantagens do programa de exercício domiciliar comparada aos programas tradicionais de reabilitação.

Quadro 2 | Vantagens e desvantagens da reabilitação cardiopulmonar domiciliar.

\begin{tabular}{|ll|}
\hline \multicolumn{1}{|c|}{ VANTAGENS } & \multicolumn{1}{c|}{ DESVANTAGENS } \\
\hline Reduz atraso para admissão na RP & Menor interação social \\
Capacidade "ilimitada" de pacientes & Segurança com pacientes de alto risco \\
Horário flexível, calendário conveniente & Dificuldades em realizar avaliações completas \\
Integração com outros afazeres domiciliares & Dificuldades na monitorização \\
\hline
\end{tabular}

Embora a reabilitação cardiopulmonar tradicionalmente seja realizada em ambulatórios ou clínicas com atendimento supervisionado, há evidências que sugerem que o treinamento físico domiciliar também pode ser eficaz. O exercício domiciliar pode desencadear efeitos similares do treinamento físico ambulatorial em pacientes com DPOC. A reabilitação cardiopulmonar domiciliar se mostrou eficaz e segura, com bons resultados principalmente no teste de caminhada de 6 minutos e nos níveis de ansiedade e depressão ${ }^{36}$.

O programa de reabilitação domiciliar, assim como as demais modalidades de RP, inclui a avaliação e o programa de intervenção dos pacientes. O programa de reabilitação deve incluir orientações para que o paciente realize o seu próprio auto-manejo e um programa de exercícios, semelhantes aos exercícios da reabilitação cardiopulmonar ambulatorial, e que incluem exercícios aeróbicos, de fortalecimento muscular, flexibilidade e equilíbrio.

\section{Considerações finais}

A reabilitação de doentes críticos acometidos por COVID-19 após alta hospitalar é de fundamental importância, especialmente naqueles que evoluíram com o quadro grave da doença, e que necessitaram de internação em UTI. A adequada avaliação para mensuração do impacto na funcionalidade irá fornecer as informações necessárias para uma reabilitação indivdualizada e eficaz.

Este é o posicionamento da ASSOBRAFIR em relação a atuação do fisioterapeuta na avaliação e intervenção para a reabilitação cardiopulmonar de pacientes recuperados da COVID-19. Esperamos, com isso, contribuir para a orientação e esclarecimento dos fisioterapeutas neste momento de incertezas. A ASSOBRAFIR está atenta à evolução dos acontecimentos e sempre que identificar necessidade emitirá nova comunicação.

Atualizado em 05 de julho de 2020. 


\section{Referências}

1. Choon-Huat Koh G, Hoenig H. How Should the Rehabilitation Community Prepare for 2019nCoV? Arch Phys Med Rehabil. 2020 Jun;101(6):1068-1071. doi: 10.1016/j.apmr.2020.03.003. Epub 2020 Mar 16.

2. Spruit MA, Holland AE, Singh SJ, Troosters T. Report of an ad-hoc international task force to develop an expert-based opinion on early and short-term rehabilitative interventions (after the acute hospital setting) in COVID-19 survivors (version April 3, 2020). [Lausanne: European Respiratory Society; 2020].182 Available from: https://ers.box.com/s/npzkvigtl4w3pb0vbsth4y0fxe7ae9z9

3. Thomas P, Baldwin C, Bissett B, Boden I, Gosselink R, Granger CL, et al. Physiotherapy management for COVID-19 in the acute hospital setting: clinical practice recommendations. J Physiother. 2020 Apr;66(2):73-82. doi: 10.1016/j.jphys.2020.03.011. Epub 2020 Mar 30.

4. Wang L, He W, Yu X, Hu D, Bao M, Liu H, et al. Coronavirus disease 2019 in elderly patients: Characteristics and prognostic factors based on 4-week follow-up. J Infect. 2020 Jun;80(6):639-645. doi: 10.1016/j.jinf.2020.03.019. Epub 2020 Mar 30.

5. Du R-H, Liang L-R, Yang C-Q, Wang W, Cao T-Z, Li M, et al. Predictors of mortality for patients with COVID-19 pneumonia caused by SARS-CoV-2: a prospective cohort study. Eur Respir J. 2020 May 7;55(5):2000524. doi: 10.1183/13993003.00524-2020. Print 2020 May.

6. Wu Z, McGoogan JM. Characteristics of and Important Lessons From the Coronavirus Disease 2019 (COVID-19) Outbreak in China. JAMA. 2020 Feb 24. doi: 10.1001/jama.2020.2648. Online ahead of print.

7. Grasselli G, Zangrillo A, Zanella A, Antonelli M, Cabrini L, Castelli A, et al. Baseline Characteristics and Outcomes of 1591 Patients Infected With SARS-CoV-2 Admitted to ICUs of the Lombardy Region, Italy. JAMA. 2020 Apr 6;323(16):1574-1581. doi: 10.1001/jama.2020.5394. Online ahead of print.

8. Richardson S, Hirsch JS, Narasimhan M, Crawford JM, McGinn T, Davidson KW, et al. Presenting Characteristics, Comorbidities, and Outcomes Among 5700 Patients Hospitalized With COVID-19 in the New York City Area. JAMA. 2020 Apr 22;323(20):2052-2059. doi: 10.1001/jama.2020.6775. Online ahead of print.

9. Ye Z, Zhang Y, Wang Y, Huang Z, Song B. Chest CT manifestations of new coronavirus disease 2019 (COVID-19): a pictorial review. Eur Radiol. 2020 Aug;30(8):4381-4389. doi: 10.1007/s00330020-06801-0. Epub 2020 Mar 19.

10. da Costa CC, de Azeredo Lermen C, Colombo C, Canterle DB, Machado MLL, Kessler A, et al. Effect of a Pulmonary Rehabilitation Program on the levels of anxiety and depression and on the quality of life of patients with chronic obstructive pulmonary disease. Rev Port Pneumol. 2014 NovDec;20(6):299-304. doi: 10.1016/j.rppneu.2014.03.007. Epub 2014 May 27.

11. Wang D, Hu B, Hu C, Zhu F, Liu X, Zhang J, et al. Clinical Characteristics of 138 Hospitalized Patients With 2019 Novel Coronavirus-Infected Pneumonia in Wuhan, China. JAMA. 2020 Feb 7;323(11):1061-1069. doi: 10.1001/jama.2020.1585. Online ahead of print.

12. Madjid M, Safavi-Naeini P, Solomon SD, Vardeny O. Potential Effects of Coronaviruses on the Cardiovascular System. JAMA Cardiol. 2020 Mar 27. doi: 10.1001/jamacardio.2020.1286. Online ahead of print. 
13. Arentz M, Yim E, Klaff L, Lokhandwala S, Riedo FX, Chong M, et al. Characteristics and Outcomes of 21 Critically Ill Patients With COVID-19 in Washington State. JAMA. 2020 Mar 19;323(16):16124. doi: 10.1001/jama.2020.4326. Online ahead of print.

14. Du R-H, Liu L-M, Yin W, Wang W, Guan L-L, Yuan M-L, et al. Hospitalization and Critical Care of 109 Decedents with COVID-19 Pneumonia in Wuhan, China. Ann Am Thorac Soc. 2020 Jul;17(7):839-846. doi: 10.1513/AnnalsATS.202003-225OC.

15. Guo T, Fan Y, Chen M, Wu X, Zhang L, He T, et al. Cardiovascular Implications of Fatal Outcomes of Patients With Coronavirus Disease 2019 (COVID-19). JAMA Cardiol. 2020 Mar 27;5(7):1-8. doi: 10.1001/jamacardio.2020.1017. Online ahead of print.

16. Liu K, Zhang W, Yang Y, Zhang J, Li Y, Chen Y. Respiratory rehabilitation in elderly patients with COVID-19: A randomized controlled study. Complement Ther Clin Pract. 2020 May;39:101166. doi: 10.1016/j.ctcp.2020.101166. Epub 2020 Apr 1.

17. Latronico N, Gosselink R. A guided approach to diagnose severe muscle weakness in the intensive care unit. Rev Bras Ter Intensiva. Jul-Sep 2015;27(3):199-201. doi: 10.5935/0103-507X.20150036. Epub 2015 Sep 15.

18. Bissett B, Leditschke IA, Green M, Marzano V, Collins S, van Haren F. Inspiratory muscle training for intensive care patients: A multidisciplinary practical guide for clinicians. Aust Crit Care. 2019 May;32(3):249-55. doi: 10.1016/j.aucc.2018.06.001. Epub 2018 Jul 11.

19. Zhou F, Yu T, Du R, Fan G, Liu Y, Liu Z, et al. Clinical course and risk factors for mortality of adult inpatients with COVID-19 in Wuhan, China: a retrospective cohort study. Lancet. 2020 Mar 28;395(10229):1054-1062. doi: 10.1016/S0140-6736(20)30566-3. Epub 2020 Mar 11.

20. Matte DL, Cacau L de AP, Reis LF da F, Assis MC. Recomendações sobre o uso de equipamentos de proteção individual (EPIs) no ambiente hospitalar e prevenção de transmissão cruzada na COVID-19 [Internet]. São Paulo: ASSOBRAFIR; 2020. Available from: https://assobrafir.com.br/ wp-content/uploads/2020/04/ASSOBRAFIR-COVID-19-EPIs 2020.04.15.pdf.

21. Mo X, Jian W, Su Z, Chen M, Peng H, Peng P, et al. Abnormal pulmonary function in COVID-19 patients at time of hospital discharge. Eur Respir J. 2020 Jun 18;55(6):2001217. doi: 10.1183/13993003.01217-2020. Print 2020 Jun.

22. Klok FA, Boon GJAM, Barco S, Endres M, Geelhoed JJM, Knauss S, et al. The Post-COVID-19 Functional Status scale: a tool to measure functional status over time after COVID-19. Eur Respir J. 2020 Jul 2;56(1):2001494. doi: 10.1183/13993003.01494-2020. Print 2020 Jul.

23. Holland AE, Spruit MA, Troosters T, Puhan MA, Pepin V, Saey D, et al. An official European Respiratory Society/ American Thoracic Society technical standard: field walking tests in chronic respiratory disease. Eur Respir J. 2014 Dec;44(6):1428-46. doi: 10.1183/09031936.00150314. Epub 2014 Oct 30.

24. Pitta F, Troosters T, Probst VS, Langer D, Decramer M, Gosselink R. Are patients with COPD more active after pulmonary rehabilitation? Chest. 2008 Aug;134(2):273-280. doi: 10.1378/chest.07-2655. Epub 2008 Apr 10.

25. Demeyer H, Burtin C, Hornikx M, Camillo CA, Van Remoortel H, Langer D, et al. The Minimal Important Difference in Physical Activity in Patients with COPD. PLoS One. 2016 Apr 28;11(4):e0154587. doi: 10.1371/journal.pone.0154587. eCollection 2016. 
26. Watz H, Pitta F, Rochester CL, Garcia-Aymerich J, ZuWallack R, Troosters T, et al. An official European Respiratory Society statement on physical activity in COPD. Eur Respir J. 2014 Dec;44(6):1521-37. doi: 10.1183/09031936.00046814. Epub 2014 Oct 30.

27. Karloh M, Araujo CLP, Gulart AA, Reis CM, Steidle LJM, Mayer AF. The Glittre-ADL test reflects functional performance measured by physical activities of daily living in patients with chronic obstructive pulmonary disease. Braz J Phys Ther. 2016 Apr 8;20(3):223-30. doi: 10.1590/ bjpt-rbf.2014.0155.

28. Sant'Anna T, Donária L, Furlanetto KC, Morakami F, Rodrigues A, Grosskreutz T, et al. Development, Validity and Reliability of the Londrina Activities of Daily Living Protocol for Subjects With COPD. Respir Care. 2017 Mar;62(3):288-297. doi: 10.4187/respcare.05058. Epub 2017 Jan 31.

29. Marra A, Pandharipande PP, Girard TD, Patel MB, Hughes CG, Jackson JC, et al. Co-Occurrence of Post-Intensive Care Syndrome Problems Among 406 Survivors of Critical Illness. Crit Care Med. 2018 Sep;46(9):1393-1401. doi: 10.1097/CCM.0000000000003218.

30. Kitsiou S, Paré G, Jaana M. Effects of Home Telemonitoring Interventions on Patients With Chronic Heart Failure: An Overview of Systematic Reviews. J Med Internet Res. 2015 Mar 12;17(3):e63. doi: 10.2196/jmir.4174.

31. Chen S-Y, Chang Y-H, Hsu H-C, Lee Y-J, Hung Y-J, Hsieh C-H. One-Year Efficacy and Safety of the Telehealth System in Poorly Controlled Type 2 Diabetic Patients Receiving Insulin Therapy. Telemed J E Health. 2011 Nov;17(9):683-7. doi: 10.1089/tmj.2011.0020. Epub 2011 Sep 1.

32. Vieira DS, Maltais F, Bourbeau J. Home-based pulmonary rehabilitation in chronic obstructive pulmonary disease patients. Curr Opin Pulm Med. 2010 Mar;16(2):134-43. doi: 10.1097/ MCP.0b013e32833642f2.

33. de Souza Y, da Silva KM, Condesso D, Figueira B, Noronha Filho AJ, Rufino R, et al. Use of a HomeBased Manual as Part of a Pulmonary Rehabilitation Program. Respir Care. 2018 Dec;63(12):14851491. doi: 10.4187/respcare.05656. Epub 2018 Aug 7.

34. Leal L, Gonçalves D, Azevedo J, Mazeika PV, Mendonça W, Reis F, et al. Use of a simple telecoaching pulmonary rehabilitation protocol for COPD patients. Eur Respir J. 2019; 54(Suppl. 63). doi: 10.1183/13993003.congress-2019.PA1260

35. Demeyer H, Louvaris Z, Frei A, Rabinovich RA, de Jong C, Gimeno-Santos E, et al. Physical activity is increased by a 12 -week semiautomated telecoaching programme in patients with COPD: a multicentre randomised controlled trial. Thorax. 2017 May;72(5):415-423. doi: 10.1136/ thoraxjnl-2016-209026. Epub 2017 Jan 30.

36. Spruit MA, Singh SJ, Garvey C, ZuWallack R, Nici L, Rochester C, et al. An official American Thoracic Society/European Respiratory Society statement: key concepts and advances in pulmonary rehabilitation. Am J Respir Crit Care Med. 2013 Oct 15;188(8):e13-64. doi: 10.1164/rccm.2013091634ST.

Submissão em: 11/07/2020

Aceite em: 15/07/2020 